\title{
APPROXIMATION OF ELLIPTIC CONTROL PROBLEMS IN MEASURE SPACES WITH SPARSE SOLUTIONS*
}

\author{
EDUARDO CASAS ${ }^{\dagger}$, CHRISTIAN CLASON $^{\ddagger}$, AND KARL KUNISCH ${ }^{\ddagger}$
}

\begin{abstract}
Optimal control problems in measure spaces governed by elliptic equations are considered for distributed and Neumann boundary control, which are known to promote sparse solutions. Optimality conditions are derived and some of the structural properties of their solutions, in particular sparsity, are discussed. A framework for their approximation is proposed which is efficient for numerical computations and for which we prove convergence and provide error estimates.
\end{abstract}

Key words. measure controls, optimal control, sparsity, elliptic partial differential equation, convergence estimates, boundary control

AMS subject classifications. 90C48, 49K20, 35J61, 35K58

DOI. $10.1137 / 110843216$

1. Introduction. This paper is dedicated to the approximation of the optimal control problem

$$
\min _{u \in \mathcal{M}(\Omega)} J(u)=\frac{1}{2}\left\|y-y_{d}\right\|_{L^{2}(\Omega)}^{2}+\alpha\|u\|_{\mathcal{M}(\Omega)}
$$

where $y$ is the unique solution to the Dirichlet problem

$$
\left\{\begin{aligned}
-\Delta y+c_{0} y=u & \text { in } \Omega, \\
y & =0 \quad \text { on } \Gamma
\end{aligned}\right.
$$

with $c_{0} \in L^{\infty}(\Omega)$ and $c_{0} \geq 0$. We assume that $\alpha>0, y_{d} \in L^{2}(\Omega)$, and $\Omega$ is a bounded domain in $\mathbb{R}^{n}, n=2$ or 3 , which is supposed to either be convex or have a $C^{1,1}$ boundary $\Gamma$. The controls are taken in the space of regular Borel measures $\mathcal{M}(\Omega)$. As usual, $\mathcal{M}(\Omega)$ is identified by the Riesz theorem with the dual space of $C_{0}(\Omega)$ - consisting of the continuous functions in $\bar{\Omega}$ vanishing on $\Gamma$ - endowed with the norm

$$
\|u\|_{\mathcal{M}(\Omega)}=\sup _{\|z\|_{C_{0}(\Omega)} \leq 1}\langle u, z\rangle=\sup _{\|z\|_{C_{0}(\Omega)} \leq 1} \int_{\Omega} z(x) d u
$$

which is equivalent to the total variation of $u$.

It has been observed that the use of measures leads to optimal controls which are sparse. This is relevant for many applications in distributed parameter control; see [6]. Moreover, the support of the optimal control provides information on the optimal

\footnotetext{
*Received by the editors August 2, 2011; accepted for publication (in revised form) March 16, 2012; published electronically July 3, 2012.

http://www.siam.org/journals/sicon/50-4/84321.html

${ }_{\dagger}^{\dagger}$ Departmento de Matemática Aplicada y Ciencias de la Computación, E.T.S.I. Industriales y de Telecomunicación, Universidad de Cantabria, 39005 Santander, Spain (eduardo.casas@unican.es). This author was supported by Spanish Ministerio de Ciencia e Innovación under projects MTM200804206 and "Ingenio Mathematica (i-MATH)" CSD2006-00032 (Consolider Ingenio 2010).

${ }^{\ddagger}$ Institute for Mathematics and Scientific Computing, University of Graz, Heinrichstrasse 36, A8010 Graz, Austria (christian.clason@uni-graz.at, karl.kunisch@uni-graz.at). These authors were supported by the Austrian Science Fund (FWF) under grant SFB F32 (SFB "Mathematical Optimization and Applications in Biomedical Sciences").
} 
placements of control actuators. Formally, the same features can be achieved by using $L^{1}(\Omega)$ control cost. In this case, however, the optimal control problem is not wellposed in the sense of a possible lack of existence of a minimizer because $L^{1}(\Omega)$ does not allow an appropriate topology for compactness arguments. Other techniques have been used to overcome this difficulty, including the use of regularization techniques or the introduction of control constraints; see, for instance, [4], [15], [16].

The focus of this paper is to give an approximation framework which, in spite of the difficulties due to the presence of measures, leads to implementable schemes for which a priori error estimates can be provided. We show that the optimal control measure can be approximated efficiently by a linear combination of Dirac measures. This is important for practical applications because it provides a way of controlling a distributed system by finitely many point actuators, giving information on where they have to be placed. A similar framework in the context of inverse problems was considered in [1].

The plan of the paper is as follows. In the next section we provide optimality conditions for (1.1) and derive some properties of the solution, in particular sparsity and actuator location. In section 3, we introduce the approximation framework and prove convergence of the discretized problems to the continuous one. Rate of convergence results are provided in section 4 . In section 5 we show that analogous results can also be obtained for Neumann control problems. Finally, the last section is devoted to numerical test problems.

2. Optimality conditions. Before establishing the optimality conditions for problem (1.1) and deducing some consequences from them, let us observe some important facts. First, given a measure $u \in \mathcal{M}(\Omega)$, we say that $y$ is a solution to (1.2) if

$$
\int_{\Omega} y A z d x=\int_{\Omega} z d u \quad \forall z \in H^{2}(\Omega) \cap H_{0}^{1}(\Omega),
$$

where $A=-\Delta+c_{0} I$. It is well known (see, for instance, [3]) that there exists a unique solution to (1.2) in the sense of (2.1). Moreover, $y \in W_{0}^{1, p}(\Omega)$ for every $1 \leq p<\frac{n}{n-1}$ and

$$
\|y\|_{W_{0}^{1, p}(\Omega)} \leq C_{p}\|u\|_{\mathcal{M}(\Omega)} .
$$

Since $W_{0}^{1, p}(\Omega) \subset L^{2}(\Omega)$ for every $\frac{2 n}{n+2} \leq p<\frac{n}{n-1}$, the cost functional is well defined on $\mathcal{M}(\Omega)$. Furthermore, the control-to-state mapping is injective, and therefore the cost functional $J$ is strictly convex. Then, it can be obtained by the standard approach that (1.1) has a unique solution; see [6] for details. Hereafter, this optimal solution will be denoted by $\bar{u}$ with an associated state $\bar{y}$. By using subdifferential calculus of convex functions and introducing the adjoint state we get the following results (see also [6], [7]).

THEOREM 2.1. There exists a unique element $\bar{\varphi} \in H^{2}(\Omega) \cap H_{0}^{1}(\Omega)$ satisfying

$$
\left\{\begin{aligned}
-\Delta \bar{\varphi}+c_{0} \bar{\varphi} & =\bar{y}-y_{d} & & \text { in } \Omega, \\
\bar{\varphi} & =0 & & \text { on } \Gamma,
\end{aligned}\right.
$$

such that

$$
\begin{gathered}
\alpha\|\bar{u}\|_{\mathcal{M}(\Omega)}+\int_{\Omega} \bar{\varphi} d \bar{u}=0, \\
\|\bar{\varphi}\|_{C_{0}(\Omega)} \begin{cases}=\alpha & \text { if } \bar{u} \neq 0, \\
\leq \alpha & \text { if } \bar{u}=0 .\end{cases}
\end{gathered}
$$


Proof. By standard arguments from Lagrange multiplier theory and the Sobolev embedding theorem, we deduce the existence of a $\lambda \in C_{0}(\Omega)$ with

$$
\lambda \in \partial\|\cdot\|_{\mathcal{M}(\Omega)}(\bar{u}) \quad \text { and } \quad \alpha \lambda=-\bar{\varphi} .
$$

By the definition of the convex subdifferential, the first inclusion is equivalent to

$$
\langle\lambda, u-\bar{u}\rangle+\|\bar{u}\|_{\mathcal{M}(\Omega)} \leq\|u\|_{\mathcal{M}(\Omega)}
$$

for all $u \in \mathcal{M}(\Omega)$. Taking $u=2 \bar{u}$ and $u=0$, respectively, we obtain the two inequalities

$$
\langle\lambda, \bar{u}\rangle \leq\|\bar{u}\|_{\mathcal{M}(\Omega)} \leq\langle\lambda, \bar{u}\rangle
$$

and hence (2.3) by the second relation of (2.5). Inserting (2.3) and $\lambda=-\frac{1}{\alpha} \bar{\varphi}$ into (2.6) yields

$$
\langle\bar{\varphi}, u\rangle \leq \alpha\|u\|_{\mathcal{M}(\Omega)}
$$

which implies (2.4).

As pointed out in [6], if we consider the Jordan decomposition of $\bar{u}=\bar{u}^{+}-\bar{u}^{-}$, then we deduce from (2.3) and (2.4) that

$$
\left\{\begin{array}{l}
\operatorname{supp}\left(\bar{u}^{+}\right) \subset\{x \in \Omega: \bar{\varphi}(x)=-\alpha\}, \\
\operatorname{supp}\left(\bar{u}^{-}\right) \subset\{x \in \Omega: \bar{\varphi}(x)=+\alpha\} .
\end{array}\right.
$$

From (2.7) we note that $\bar{u} \equiv 0$ on the set $\{x \in \Omega:|\bar{\varphi}(x)|<\alpha\}$. As the numerical results will show, the set $\{x \in \Omega:|\bar{\varphi}(x)|=\alpha\}$ is small, which yields the sparsity of $\bar{u}$. Moreover, we have the following property for the penalty parameter.

Proposition 2.2. There exists $\bar{\alpha}>0$ such that $\bar{u}=0$ for every $\alpha>\bar{\alpha}$.

Proof. Let us denote by $J_{\alpha}$ the cost functional associated to the parameter $\alpha$. Similarly, let $\left(u_{\alpha}, y_{\alpha}, \varphi_{\alpha}\right)$ denote the solution to the corresponding optimality system. For each $\alpha>0$ the following inequalities hold:

$$
\frac{1}{2}\left\|y_{\alpha}-y_{d}\right\|_{L^{2}(\Omega)}^{2} \leq J_{\alpha}\left(u_{\alpha}\right) \leq J_{\alpha}(0)=\frac{1}{2}\left\|y_{d}\right\|_{L^{2}(\Omega)}^{2}
$$

Consequently,

$$
\left\|y_{\alpha}-y_{d}\right\|_{L^{2}(\Omega)} \leq\left\|y_{d}\right\|_{L^{2}(\Omega)} \quad \forall \alpha>0 .
$$

From the adjoint state equation and the embedding of $H^{2}(\Omega) \cap H_{0}^{1}(\Omega) \hookrightarrow C_{0}(\Omega)$, we deduce the existence of a constant $C>0$ such that

$$
\left\|\varphi_{\alpha}\right\|_{C_{0}(\Omega)} \leq C\left\|y_{\alpha}-y_{d}\right\|_{L^{2}(\Omega)} \leq C\left\|y_{d}\right\|_{L^{2}(\Omega)} .
$$

Setting $\bar{\alpha}=C\left\|y_{d}\right\|_{L^{2}(\Omega)}$, we obtain from the above inequality and (2.4) that $u_{\alpha}=0$ for every $\alpha>\bar{\alpha}$.

In the case where we consider the observation of the state only in a subset $\omega_{y} \subset \Omega$, we have the following property of the support of the optimal control.

Proposition 2.3. Let $\omega_{y}$ be an open subset of $\Omega$ such that $\Omega \backslash \omega_{y}$ is connected and consider the functional

$$
J_{\omega_{y}}(u)=\frac{1}{2}\left\|y-y_{d}\right\|_{L^{2}\left(\omega_{y}\right)}^{2}+\alpha\|u\|_{\mathcal{M}(\Omega)} .
$$

Then the associated optimal control $\bar{u}$ satisfies $\operatorname{supp}(\bar{u}) \subset \bar{\omega}_{y}$.

Copyright (c) by SIAM. Unauthorized reproduction of this article is prohibited. 

by

Proof. For the functional under consideration, the adjoint state equation is given

$$
\left\{\begin{aligned}
-\Delta \bar{\varphi}+c_{0} \bar{\varphi} & =\left(\bar{y}-y_{d}\right) \chi_{\omega_{y}} & & \text { in } \Omega, \\
\bar{\varphi} & =0 & & \text { on } \Gamma,
\end{aligned}\right.
$$

where $\chi_{\omega_{y}}$ is the indicator function of $\omega_{y}$. Applying the maximum principle to the problem

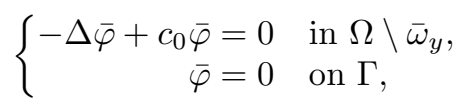

we deduce that $\bar{\varphi}$ is identically zero in $\Omega \backslash \bar{\omega}_{y}$ or

$$
\min _{x^{\prime} \in \partial \omega_{y}} \bar{\varphi}\left(x^{\prime}\right)<\bar{\varphi}(x)<\max _{x^{\prime} \in \partial \omega_{y}} \bar{\varphi}\left(x^{\prime}\right) \quad \forall x \in \Omega \backslash \bar{\omega}_{y} .
$$

In both cases the equality (2.4) can only be achieved in $\bar{\omega}_{y}$; therefore (2.7) implies the claim of the proposition.

Let us close this section by pointing out that the results of our paper can also be adapted to the situation where the control domain is a priori restricted to a strict subdomain $\omega_{u}$ of $\Omega$, and the controls are restricted to be nonnegative (cf. [7]).

3. Approximation of (1.1). In this section $\Omega$ will be assumed to be convex. We consider a nodal basis finite element approximation of (1.1). Associated with a parameter $h$ we consider a family of triangulations $\left\{\mathcal{T}_{h}\right\}_{h>0}$ of $\bar{\Omega}$. To every element $T \in \mathcal{T}_{h}$ we assign two parameters $\rho(T)$ and $\sigma(T)$, where $\rho(T)$ denotes the diameter of $T$ and $\sigma(T)$ is the diameter of the biggest ball contained in $T$. The size of the grid is given by $h=\max _{T \in \mathcal{T}_{h}} \rho(T)$. The following usual regularity assumptions on the triangulation are assumed:

(i) There exist two positive constants $\rho$ and $\sigma$ such that

$$
\frac{\rho(T)}{\sigma(T)} \leq \sigma \quad \text { and } \quad \frac{h}{\rho(T)} \leq \rho
$$

hold for every $T \in \mathcal{T}_{h}$ and all $h>0$.

(ii) Let us set $\bar{\Omega}_{h}=\cup_{T \in \mathcal{T}_{h}} T$ with $\Omega_{h}$ and $\Gamma_{h}$ its interior and boundary, respectively. We assume that the vertices of $\mathcal{T}_{h}$ placed on the boundary $\Gamma_{h}$ are also points of $\Gamma$. From [13, inequality (5.2.19)] we know

$$
\left|\Omega \backslash \Omega_{h}\right| \leq C h^{2}
$$

where $|\cdot|$ denotes the Lebesgue measure.

Associated to these triangulations we define the space

$$
Y_{h}=\left\{y_{h} \in C_{0}(\Omega): y_{h \mid T} \in \mathcal{P}_{1} \text { for every } T \in \mathcal{T}_{h} \text {, and } y_{h}=0 \text { in } \bar{\Omega} \backslash \Omega_{h}\right\},
$$

where $\mathcal{P}_{1}$ is the space formed by the polynomials of degree less than or equal to one. For every $u \in \mathcal{M}(\Omega)$, we denote by $y_{h}$ the unique element of $Y_{h}$ satisfying

$$
a\left(y_{h}, z_{h}\right)=\int_{\Omega_{h}} z_{h} d u \quad \forall z_{h} \in Y_{h}
$$


where $a: H^{1}(\Omega) \times H^{1}(\Omega) \rightarrow \mathbb{R}$ is the bilinear form associated to the operator $A$, i.e.,

$$
a(y, z)=\int_{\Omega}\left[\nabla y(x) \nabla z(x)+c_{0}(x) y(x) z(x)\right] d x .
$$

The approximation of the optimal control problem (1.1) is defined as

$$
\left(\mathrm{P}_{h}\right) \quad \min _{u \in \mathcal{M}(\Omega)} J_{h}\left(u_{h}\right)=\frac{1}{2}\left\|y_{h}-y_{d}\right\|_{L^{2}\left(\Omega_{h}\right)}^{2}+\alpha\|u\|_{\mathcal{M}(\Omega)},
$$

where $y_{h}$ is the solution to (3.2).

Since we have not discretized the control space, this approach is related to the variational discretization method introduced in [8]. Below we will show that among all the solutions to (3.3) there is a unique one which is a finite linear combination of Dirac measures concentrated in the interior vertices of the triangulation, leading to a simple numerical implementation.

Before any discussion of the solutions to problem (3.3), let us introduce some additional notation. Hereafter we will denote by $\left\{x_{j}\right\}_{j=1}^{N(h)}$ the interior nodes of the triangulation $\mathcal{T}_{h}$. Associated to these nodes we consider the nodal basis of $Y_{h}$ given by the functions $\left\{e_{j}\right\}_{j=1}^{N(h)}$ such that $e_{j}\left(x_{i}\right)=\delta_{i j}$ for every $1 \leq i, j \leq N(h)$. Then every element $y_{h}$ of $Y_{h}$ can be written in the form

$$
y_{h}=\sum_{j=1}^{N(h)} y_{j} e_{j}, \quad \text { where } \quad y_{j}=y_{h}\left(x_{j}\right), \quad 1 \leq j \leq N(h) .
$$

We also consider the space

$$
D_{h}=\left\{u_{h} \in \mathcal{M}(\Omega): u_{h}=\sum_{j=1}^{N(h)} \lambda_{j} \delta_{x_{j}}, \text { where }\left\{\lambda_{j}\right\}_{j=1}^{N(h)} \subset \mathbb{R}\right\} .
$$

Above $\delta_{x_{j}}$ denotes the Dirac measure centered at the node $x_{j}$. It is obvious that $D_{h}$ can be identified with the dual of $Y_{h}$ through the duality relation

$$
\left\langle u_{h}, y_{h}\right\rangle=\sum_{j=1}^{N(h)} \lambda_{j} y_{j}
$$

Now, we define the linear operators $\Pi_{h}: C_{0}(\Omega) \rightarrow Y_{h}$ and $\Lambda_{h}: \mathcal{M}(\Omega) \rightarrow D_{h}$ by

$$
\Pi_{h} y=\sum_{j=1}^{N(h)} y\left(x_{j}\right) e_{j} \quad \text { and } \quad \Lambda_{h} u=\sum_{j=1}^{N(h)}\left\langle u, e_{j}\right\rangle \delta_{x_{j}} .
$$

The operator $\Pi_{h}$ is the nodal interpolation operator for $Y_{h}$, and we have the following result concerning the operator $\Lambda_{h}$.

THEOREM 3.1. The following properties hold.

1. For every $u \in \mathcal{M}(\Omega)$ and every $z \in C_{0}(\Omega)$ and $z_{h} \in Y_{h}$ we have

$$
\begin{aligned}
\left\langle u, z_{h}\right\rangle & =\left\langle\Lambda_{h} u, z_{h}\right\rangle, \\
\left\langle u, \Pi_{h} z\right\rangle & =\left\langle\Lambda_{h} u, z\right\rangle .
\end{aligned}
$$

Copyright $@$ by SIAM. Unauthorized reproduction of this article is prohibited. 
2. For every $u \in \mathcal{M}(\Omega)$ we have

$$
\begin{aligned}
& \left\|\Lambda_{h} u\right\|_{\mathcal{M}(\Omega)} \leq\|u\|_{\mathcal{M}(\Omega)}, \\
& \Lambda_{h} u \stackrel{*}{\rightarrow} u \text { in } \mathcal{M}(\Omega) \text { and }\left\|\Lambda_{h} u\right\|_{\mathcal{M}(\Omega)} \rightarrow\|u\|_{\mathcal{M}(\Omega)} .
\end{aligned}
$$

3. There exist a constant $C>0$ such that for every $u \in \mathcal{M}(\Omega)$

$$
\begin{aligned}
\left\|u-\Lambda_{h} u\right\|_{W^{-1, p}(\Omega)} & \leq C h^{1-n / p^{\prime}}\|u\|_{\mathcal{M}(\Omega)}, \quad 1<p<\frac{n}{n-1}, \\
\left\|u-\Lambda_{h} u\right\|_{\left(W_{0}^{1, \infty}(\Omega)\right)^{*}} & \leq C h\|u\|_{\mathcal{M}(\Omega)},
\end{aligned}
$$

where $p^{\prime}$ is the conjugate of $p$.

4. Given $u \in \mathcal{M}(\Omega)$, and let $y_{h}$ and $\tilde{y}_{h}$ be the solutions to (3.2) associated to the controls $u$ and $\Lambda_{h} u$, respectively. Then the equality $y_{h}=\tilde{y}_{h}$ holds.

Proof. For $z_{h}=\sum_{j=1}^{N(h)} z_{j} e_{j}$ we have

$$
\left\langle u, z_{h}\right\rangle=\sum_{j=1}^{N(h)} z_{j}\left\langle u, e_{j}\right\rangle=\sum_{j=1}^{N(h)}\left\langle u, e_{j}\right\rangle\left\langle\delta_{x_{j}}, z_{h}\right\rangle=\left\langle\Lambda_{h} u, z_{h}\right\rangle,
$$

which proves (3.4). For (3.5) we proceed as follows:

$$
\left\langle u, \Pi_{h} z\right\rangle=\sum_{j=1}^{N(h)} z\left(x_{j}\right)\left\langle u, e_{j}\right\rangle=\sum_{j=1}^{N(h)}\left\langle u, e_{j}\right\rangle\left\langle\delta_{x_{j}}, z\right\rangle=\left\langle\Lambda_{h} u, z\right\rangle .
$$

To verify (3.6) we introduce the function $s_{h} \in Y_{h}$ by

$$
s_{h}=\sum_{j=1}^{N(h)} s_{j} e_{j}, \quad \text { with } \quad s_{j}= \begin{cases}+1 & \text { if }\left\langle u, e_{j}\right\rangle>0 \\ -1 & \text { if }\left\langle u, e_{j}\right\rangle<0 \\ 0 & \text { otherwise }\end{cases}
$$

Then we have

$$
\begin{aligned}
\left\|\Lambda_{h} u\right\|_{\mathcal{M}(\Omega)} & =\sum_{j=1}^{N(h)}\left|\left\langle u, e_{j}\right\rangle\right|=\sum_{j=1}^{N(h)} s_{j}\left\langle u, e_{j}\right\rangle=\left\langle u, s_{h}\right\rangle \leq\|u\|_{\mathcal{M}(\Omega)}\left\|s_{h}\right\|_{C_{0}(\Omega)} \\
& =\|u\|_{\mathcal{M}(\Omega) .}
\end{aligned}
$$

Let us prove (3.7). Since $\left\{\Lambda_{h} u\right\}_{h>0}$ is bounded in $\mathcal{M}(\Omega)$ there exists a subsequence, denoted in the same way, such that $\Lambda_{h} u \stackrel{*}{\rightarrow} v$ in $\mathcal{M}(\Omega)$. From (3.4) we get that

$$
\left\langle v, e_{j}\right\rangle=\lim _{h \rightarrow 0}\left\langle\Lambda_{h} u, e_{j}\right\rangle=\left\langle u, e_{j}\right\rangle \quad \forall 1 \leq j \leq N(h),
$$

which implies that $\left\langle v, z_{h}\right\rangle=\left\langle u, z_{h}\right\rangle$ for every $z_{h} \in Y_{h}$. Hence, for every $z \in C_{0}(\Omega)$

$$
\langle v, z\rangle=\lim _{h \rightarrow 0}\left\langle v, \Pi_{h} z\right\rangle=\lim _{h \rightarrow 0}\left\langle u, \Pi_{h} z\right\rangle=\langle u, z\rangle,
$$

therefore $u=v$. Since any subsequence converges to $u$, the whole sequence converges

Copyright $@$ by SIAM. Unauthorized reproduction of this article is prohibited. 
to $u$ weakly* in $\mathcal{M}(\Omega)$. From this convergence and (3.6) we obtain

$$
\|u\|_{\mathcal{M}(\Omega)} \leq \liminf _{h \rightarrow 0}\left\|\Lambda_{h} u\right\|_{\mathcal{M}(\Omega)} \leq \limsup _{h \rightarrow 0}\left\|\Lambda_{h} u\right\|_{\mathcal{M}(\Omega)} \leq\|u\|_{\mathcal{M}(\Omega)},
$$

and consequently (3.7) holds.

To prove (3.8) we take an arbitrary element $z \in W_{0}^{1, p^{\prime}}(\Omega)$ with $1 \leq p<\frac{n}{n-1}$. Using (3.5) and the well known interpolation error estimates in Sobolev spaces (see, for instance, [5, Chapter 3]) we obtain

$$
\begin{aligned}
\left\langle u-\Lambda_{h} u, z\right\rangle=\left\langle u, z-\Pi_{h} z\right\rangle & \leq\|u\|_{\mathcal{M}(\Omega)}\left\|z-\Pi_{h} z\right\|_{C_{0}(\Omega)} \\
& \leq C h^{1-n / p^{\prime}}\|u\|_{\mathcal{M}(\Omega)}\|z\|_{W_{0}^{1, p^{\prime}}(\Omega)} .
\end{aligned}
$$

Since $W^{-1, p}(\Omega)$ is the dual of $W_{0}^{1, p^{\prime}}(\Omega)$ for $1<p<\frac{n}{n-1},(3.8)$ follows from the above inequalities. For $p=1$, we have $p^{\prime}=\infty$ and the above inequality can be expressed as

$$
\left\langle u-\Lambda_{h} u, z\right\rangle \leq C h\|u\|_{\mathcal{M}(\Omega)}\|z\|_{W_{0}^{1, \infty}(\Omega)} \quad \forall z \in W_{0}^{1, \infty}(\Omega) .
$$

Since $W^{-1,1}(\Omega)$ is not the dual space of $W_{0}^{1, \infty}(\Omega)$, from this inequality we only get (3.9).

The last statement of the theorem is an immediate consequence of (3.4).

Now, we turn to the study of (3.3). First, we observe that analogously to $J$, the functional $J_{h}$ is convex. However, it is not strictly convex. This is a consequence of the noninjectivity of the control-to-discrete-state mapping and the nonstrict convexity of the norm of $\mathcal{M}(\Omega)$. Although the existence of a solution can be proved in the same way as for the problem (1.1), we cannot claim its uniqueness. Nevertheless, if $\tilde{u}_{h}$ is a solution to (3.3) and we take $\bar{u}_{h}=\Lambda_{h} \tilde{u}_{h}$, then statement 4 of Theorem 3.1 and the inequality (3.6) imply that $J_{h}\left(\bar{u}_{h}\right) \leq J_{h}\left(\tilde{u}_{h}\right)$, and hence $\bar{u}_{h}$ is also a solution to (3.3). Since for $u_{h} \in D_{h}$, the mapping $u_{h} \mapsto y_{h}\left(u_{h}\right)$, the solution to (3.2) for $u=u_{h}$, is linear, injective, and $\operatorname{dim} D_{h}=\operatorname{dim} Y_{h}$, this mapping is bijective. Therefore, the cost functional $J_{h}$ is strictly convex on $D_{h}$, and hence (3.3) has a unique solution in $D_{h}$, which will be denoted by $\bar{u}_{h}$ hereafter. We summarize this discussion in the following theorem.

Theorem 3.2. Problem (3.3) admits at least one solution. Among them there exists a unique one $\bar{u}_{h}$ belonging to $D_{h}$. Moreover, any other solution $\tilde{u}_{h} \in \mathcal{M}(\Omega)$ of (3.3) satisfies that $\Lambda_{h} \tilde{u}_{h}=\bar{u}_{h}$.

Remark 3.3. The fact that (3.3) has exactly one solution in $D_{h}$ is of practical interest. Indeed, recall that as an element of $D_{h}, \bar{u}_{h}$ has a unique representation of the form

$$
\bar{u}_{h}=\sum_{j=1}^{N(h)} \bar{\lambda}_{j} \delta_{x_{j}} .
$$

Then, the numerical computation of $\bar{u}_{h}$ is reduced to the computation of the coefficients $\left\{\bar{\lambda}_{j}\right\}_{j=1}^{N(h)}$.

Remark 3.4. All results remain valid for Lagrange elements of arbitrary degree, where the $x_{j}$ should be taken as the nodes associated with the degrees of freedom (which no longer need to correspond to vertices of the triangulation, e.g., vertices and edge midpoints for quadratic elements).

We finish this section by proving the convergence of the solutions in $D_{h}$ to problems (3.3) to the solution to (1.1). 
Theorem 3.5. For every $h>0$, let $\bar{u}_{h}$ be the unique solution to (3.3) belonging to $D_{h}$ and let $\bar{u}$ be the solution to (1.1). Then the following convergence properties hold for $h \rightarrow 0$ :

$$
\begin{aligned}
\bar{u}_{h} & \stackrel{*}{\rightarrow} \quad \text { in } \mathcal{M}(\Omega), \\
\left\|\bar{u}_{h}\right\|_{\mathcal{M}(\Omega)} & \rightarrow\|\bar{u}\|_{\mathcal{M}(\Omega)}, \\
\left\|\bar{y}-\bar{y}_{h}\right\|_{L^{2}(\Omega)} & \rightarrow 0 \\
J_{h}\left(\bar{u}_{h}\right) & \rightarrow J(\bar{u}),
\end{aligned}
$$

where $\bar{y}$ and $\bar{y}_{h}$ are the continuous and discrete states associated to $\bar{u}$ and $\bar{u}_{h}$, respectively.

Proof. First, let us verify that

$$
u_{h} \stackrel{*}{\rightarrow} u \text { in } \mathcal{M}(\Omega) \quad \text { implies } \quad\left\|y_{h}\left(u_{h}\right)-y_{u}\right\|_{L^{2}(\Omega)} \rightarrow 0,
$$

where $y_{h}\left(u_{h}\right)$ and $y_{u}$ are the discrete and continuous states associated to the controls $u_{h}$ and $u$, respectively. From the compact embedding $\mathcal{M}(\Omega) \hookrightarrow W^{-1, p}(\Omega)$ for every $1 \leq p<\frac{n}{n-1}$, we deduce the strong converge $u_{h} \rightarrow u$ in $W^{-1, p}(\Omega)$. Let us denote by $y_{u_{h}}$ the continuous state associated to $u_{h}$. From [9] we obtain the strong convergence $y_{u_{h}} \rightarrow y_{u}$ in $W^{1, p}(\Omega)$, where we have used that the boundary $\Gamma$ is Lipschitz continuous as a consequence of the convexity of $\Omega$. Moreover, from [2] we have that $\| y_{h}\left(u_{h}\right)-$ $y_{u_{h}} \|_{L^{2}(\Omega)} \rightarrow 0$. Finally, by the triangular inequality we obtain the desired convergence.

Turning to the verification of (3.10), we observe that

$$
\alpha\left\|\bar{u}_{h}\right\|_{\mathcal{M}(\Omega)} \leq J_{h}\left(\bar{u}_{h}\right) \leq J_{h}(0)=\frac{1}{2}\left\|y_{d}\right\|_{L^{2}\left(\Omega_{h}\right)}^{2} \leq \frac{1}{2}\left\|y_{d}\right\|_{L^{2}(\Omega)}^{2},
$$

which implies the boundedness of $\left\{\bar{u}_{h}\right\}_{h>0}$ in $\mathcal{M}(\Omega)$. By taking a subsequence, we have that $\bar{u}_{h} \stackrel{*}{\rightarrow} v$ in $\mathcal{M}(\Omega)$. Then using (3.1), (3.14), the lower semicontinuity of the norm $\|\cdot\|_{\mathcal{M}(\Omega)}$, and (3.7) we get

$$
J(v) \leq \liminf _{h \rightarrow 0} J_{h}\left(\bar{u}_{h}\right) \leq \limsup _{h \rightarrow 0} J_{h}\left(\bar{u}_{h}\right) \leq \limsup _{h \rightarrow 0} J_{h}\left(\Lambda_{h} \bar{u}\right)=J(\bar{u}) .
$$

Hence $v=\bar{u}$ by the uniqueness of the solution to (1.1), and the whole sequence $\left\{\bar{u}_{h}\right\}_{h>0}$ converges weakly* to $\bar{u}$. Also, from the above inequality we get (3.13). Using again (3.14), we deduce (3.12). Finally, (3.11) follows immediately from (3.12) and (3.13).

4. Error estimates. This section is devoted to the proof of error estimates for the optimal costs as well as for the optimal states. We still require $\Omega$ to be convex and in addition we assume

$$
y_{d} \in L^{r}(\Omega) \text { with } r= \begin{cases}4 & \text { if } n=2, \\ \frac{8}{3} & \text { if } n=3 .\end{cases}
$$

As in the previous sections, we denote by $\bar{y}$ and $\bar{y}_{h}$ the continuous and discrete states associated to the optimal controls $\bar{u}$ and $\bar{u}_{h}$, respectively.

THEOREM 4.1. There exists a constant $C>0$ independent of $h$ such that

$$
\left|J(\bar{u})-J_{h}\left(\bar{u}_{h}\right)\right| \leq C h^{\kappa},
$$

where $\kappa=1$ if $n=2$ and $\kappa=1 / 2$ if $n=3$.

Copyright (C) by SIAM. Unauthorized reproduction of this article is prohibited. 
Proof. We establish some preliminary estimates. Given $u \in \mathcal{M}(\Omega)$, with associated continuous and discrete states $y$ and $y_{h}$, we know from [2] that

$$
\left\|y-y_{h}\right\|_{L^{2}\left(\Omega_{h}\right)} \leq C h^{\kappa}\|u\|_{\mathcal{M}(\Omega)}
$$

with $\kappa$ defined as in the statement of the theorem.

Taking $r$ as in (4.1) and using Hölder's inequality and (3.1), we deduce that for all $\phi \in L^{r}(\Omega)$,

$$
\|\phi\|_{L^{2}\left(\Omega \backslash \Omega_{h}\right)} \leq\|\phi\|_{L^{r}\left(\Omega \backslash \Omega_{h}\right)}\left|\Omega \backslash \Omega_{h}\right|^{\frac{r-2}{2 r}} \leq C\|\phi\|_{L^{r}\left(\Omega \backslash \Omega_{h}\right)} h^{\frac{\kappa}{2}}
$$

holds. As a consequence of (4.3) and (4.4), with $\phi=y-y_{d}$, we get

$$
\begin{aligned}
& \left|\left\|y-y_{d}\right\|_{L^{2}(\Omega)}^{2}-\left\|y_{h}-y_{d}\right\|_{L^{2}\left(\Omega_{h}\right)}^{2}\right| \\
& \quad \leq\left\|y-y_{d}\right\|_{L^{2}\left(\Omega \backslash \Omega_{h}\right)}^{2}+\left(\left\|y-y_{d}\right\|_{L^{2}\left(\Omega_{h}\right)}+\left\|y_{h}-y_{d}\right\|_{L^{2}\left(\Omega_{h}\right)}\right)\left\|y-y_{h}\right\|_{L^{2}\left(\Omega_{h}\right)} \\
& \quad \leq C\left(\left\|y-y_{d}\right\|_{L^{r}\left(\Omega \backslash \Omega_{h}\right)}^{2}+\left[\left\|y-y_{d}\right\|_{L^{2}\left(\Omega_{h}\right)}+\left\|y_{h}-y_{d}\right\|_{L^{2}\left(\Omega_{h}\right)}\right]\|u\|_{\mathcal{M}(\Omega)}\right) h^{\kappa} .
\end{aligned}
$$

Now, by the optimality of $\bar{u}$ and $\bar{u}_{h}$ we have

$$
J(\bar{u})-J_{h}(\bar{u}) \leq J(\bar{u})-J_{h}\left(\bar{u}_{h}\right) \leq J\left(\bar{u}_{h}\right)-J_{h}\left(\bar{u}_{h}\right),
$$

and hence

$$
\left|J(\bar{u})-J_{h}\left(\bar{u}_{h}\right)\right| \leq \max \left\{\left|J(\bar{u})-J_{h}(\bar{u})\right|,\left|J\left(\bar{u}_{h}\right)-J_{h}\left(\bar{u}_{h}\right)\right|\right\} .
$$

From (3.11) we deduce that $\left\{\bar{u}_{h}\right\}_{h>0}$ is bounded in $\mathcal{M}(\Omega)$. Therefore, (2.2) implies that the continuous associated states $\left\{y_{\bar{u}_{h}}\right\}_{h>0}$ are bounded in $W_{0}^{1, p}(\Omega)$ for every $1 \leq p<\frac{n}{n-1}$ and therefore also in $L^{r}(\Omega)$. We apply (4.5) with $u=\bar{u}_{h}$ and $u=\bar{u}$, respectively. Together with (4.6) this establishes (4.2).

In the following theorem we establish a rate of convergence for the states.

THEOREM 4.2. There exists a constant $C>0$ independent of $h$ such that

$$
\left\|\bar{y}-\bar{y}_{h}\right\|_{L^{2}(\Omega)} \leq C h^{\frac{\kappa}{2}}
$$

with $\kappa$ as defined in Theorem 4.1 .

Proof. Let $S: \mathcal{M}(\Omega) \rightarrow L^{2}(\Omega)$ and $S_{h}: \mathcal{M}(\Omega) \rightarrow L^{2}(\Omega)$ be the solution operators associated to (1.2) and (3.2), respectively. From (4.3) it follows that

$$
\left\|S u-S_{h} u\right\|_{L^{2}\left(\Omega_{h}\right)} \leq C h^{\kappa}\|u\|_{\mathcal{M}(\Omega)} .
$$

By the optimality of $\bar{u}$ we have for all $u \in \mathcal{M}(\Omega)$ that

$$
\left(S \bar{u}-y_{d}, S u-S \bar{u}\right)+\alpha\left[\|u\|_{\mathcal{M}(\Omega)}-\|\bar{u}\|_{\mathcal{M}(\Omega)}\right] \geq 0,
$$

where $(\cdot, \cdot)$ denotes the scalar product in $L^{2}(\Omega)$. In particular, taking $u=\bar{u}_{h}$, we get

$$
\left(S \bar{u}-y_{d}, S \bar{u}_{h}-S \bar{u}\right)+\alpha\left[\left\|\bar{u}_{h}\right\|_{\mathcal{M}(\Omega)}-\|\bar{u}\|_{\mathcal{M}(\Omega)}\right] \geq 0 .
$$

Analogously, the optimality of $\bar{u}_{h}$ implies that

$$
\left(S_{h} \bar{u}_{h}-y_{d}, S_{h} \bar{u}-S_{h} \bar{u}_{h}\right)+\alpha\left[\|\bar{u}\|_{\mathcal{M}(\Omega)}-\left\|\bar{u}_{h}\right\|_{\mathcal{M}(\Omega)}\right] \geq 0 .
$$

Copyright $@$ by SIAM. Unauthorized reproduction of this article is prohibited. 
We point out that by definition of $Y_{h}$, we have $S_{h} u=0$ in $\Omega \backslash \Omega_{h}$. Then, the scalar product above in $L^{2}(\Omega)$ coincides with that in $L^{2}\left(\Omega_{h}\right)$. Now, we rearrange terms in (4.10) as follows:

$$
\begin{aligned}
\left(S \bar{u}_{h}-y_{d}, S \bar{u}-S \bar{u}_{h}\right)+\left(S_{h} \bar{u}_{h}-S \bar{u}_{h}, S_{h} \bar{u}-S_{h} \bar{u}_{h}\right) & \\
+\left(y_{d}, S \bar{u}-S_{h} \bar{u}+S_{h} \bar{u}_{h}-S \bar{u}_{h}\right)+ & \left(S \bar{u}_{h}, S_{h} \bar{u}-S \bar{u}+S \bar{u}_{h}-S_{h} \bar{u}_{h}\right) \\
& +\alpha\left[\|\bar{u}\|_{\mathcal{M}(\Omega)}-\left\|\bar{u}_{h}\right\|_{\mathcal{M}(\Omega)}\right] \geq 0 .
\end{aligned}
$$

Now, adding (4.9) and (4.11) we obtain

$$
\begin{aligned}
\left\|S \bar{u}-S_{h} \bar{u}_{h}\right\|_{L^{2}(\Omega)}^{2}= & \left(S \bar{u}-S_{h} \bar{u}_{h}, S \bar{u}-S_{h} \bar{u}_{h}\right) \\
\leq & \left(S_{h} \bar{u}_{h}-S \bar{u}_{h}, S_{h} \bar{u}-S_{h} \bar{u}_{h}\right) \\
& +\left(y_{d}-S \bar{u}_{h}, S \bar{u}-S_{h} \bar{u}+S_{h} \bar{u}_{h}-S \bar{u}_{h}\right) .
\end{aligned}
$$

Let us estimate the right-hand terms. For the first one we apply the Cauchy-Schwarz inequality, exploit the fact $S_{h} \bar{u}-S_{h} \bar{u}_{h}=0$ in $\Omega \backslash \Omega_{h}$, and use (4.8) to deduce

$$
\left(S_{h} \bar{u}_{h}-S \bar{u}_{h}, S_{h} \bar{u}-S_{h} \bar{u}_{h}\right) \leq\left\|S_{h} \bar{u}_{h}-S \bar{u}_{h}\right\|_{L^{2}\left(\Omega_{h}\right)}\left\|S_{h} \bar{u}-S_{h} \bar{u}_{h}\right\| \leq C h^{\kappa},
$$

where we have used that $\left\{\bar{u}_{h}\right\}_{h>0},\left\{S_{h} \bar{u}\right\}_{h>0}$ and $\left\{S_{h} \bar{u}_{h}\right\}_{h>0}$ are bounded due to (3.11), (3.12), and (4.3), respectively. For the second term we use (4.4) and once again (4.8) as well as the fact that $S_{h} u=0$ in $\Omega \backslash \Omega_{h}$ to obtain

$$
\begin{aligned}
\left(y_{d}-S \bar{u}_{h}, S \bar{u}-S_{h} \bar{u}+S_{h} \bar{u}_{h}-S \bar{u}_{h}\right) \leq & \left\|y_{d}-S \bar{u}_{h}\right\|_{L^{2}\left(\Omega \backslash \Omega_{h}\right)}\left\|S\left(\bar{u}-\bar{u}_{h}\right)\right\|_{L^{2}\left(\Omega \backslash \Omega_{h}\right)} \\
& +\left\|y_{d}-S \bar{u}_{h}\right\|_{L^{2}\left(\Omega_{h}\right)}\left\|\left(S-S_{h}\right)\left(\bar{u}-\bar{u}_{h}\right)\right\|_{L^{2}\left(\Omega_{h}\right)} \\
\leq & C\left(\left\|y_{d}-S \bar{u}_{h}\right\|_{L^{r}\left(\Omega \backslash \Omega_{h}\right)}\left\|S\left(\bar{u}-\bar{u}_{h}\right)\right\|_{L^{r}\left(\Omega \backslash \Omega_{h}\right)}\right. \\
& \left.+\left\|\bar{u}-\bar{u}_{h}\right\|_{\mathcal{M}(\Omega)}\right) h^{\kappa} \leq C h^{\kappa},
\end{aligned}
$$

where we have also used that $y_{d} \in L^{r}(\Omega)$ and (2.2). Finally, (4.12), (4.13), and (4.14) prove (4.7).

Remark 4.3. Let us observe that (4.2) and (4.7) imply that

$$
\left|\|\bar{u}\|_{\mathcal{M}(\Omega)}-\left\|\bar{u}_{h}\right\|_{\mathcal{M}(\Omega)}\right| \leq C h^{\frac{\kappa}{2}}
$$

for some constant $C>0$ independent of $h$.

Remark 4.4. All the previous results remain correct for a general elliptic operator

$$
A y=-\sum_{i, j=1}^{n} \partial_{x_{j}}\left[a_{i j} \partial_{x_{i}} y\right]+a_{0} y,
$$

provided the coefficients $a_{i j}$ are Lipschitz continuous functions in $\bar{\Omega}$ and $a_{0} \geq 0$ is in $L^{\infty}(\Omega)$.

5. A Neumann control problem. In this section, we assume that the system is controlled on the boundary. The control problem is formulated as

$$
\left(\mathrm{P}_{\Gamma}\right) \quad \min _{u \in \mathcal{M}(\Gamma)} J_{\Gamma}(u)=\frac{1}{2}\left\|y-y_{d}\right\|_{L^{2}(\Omega)}^{2}+\alpha\|u\|_{\mathcal{M}(\Gamma)},
$$

Copyright (c) by SIAM. Unauthorized reproduction of this article is prohibited. 
where $y$ is the unique solution to the Neumann problem

$$
\left\{\begin{aligned}
-\Delta y+c_{0} y=f & \text { in } \Omega, \\
\partial_{\nu} y=u & \text { on } \Gamma
\end{aligned}\right.
$$

for $c_{0} \in L^{\infty}(\Omega), c_{0} \geq 0$, and $c_{0} \not \equiv 0$ and given $f \in L^{1}(\Omega)$. Here we will assume $\Omega \subset \mathbb{R}^{n}$, $n=2$ or 3 , to be convex and polyhedral. Again by the Riesz representation theorem $\mathcal{M}(\Gamma)$ is identified with the dual space of $C(\Gamma)$; see, for instance, [14, Chapter 6]. Concerning the state equation (5.1), analogously to the Dirichlet problem (1.2), we say that an element $y \in W^{1, p}(\Omega), p<\frac{n}{n-1}$, is a solution to (5.1) if

$$
\int_{\Omega} y A z d x+\int_{\Gamma} y \partial_{\nu} z d \sigma=\int_{\Omega} f z d x+\int_{\Gamma} z d u \quad \forall z \in H^{2}(\Omega) .
$$

We have the following theorem.

THEOREM 5.1. The problem (5.1) has a unique solution belonging to $W^{1, p}(\Omega)$ for every $1 \leq p<\frac{n}{n-1}$, and there exists a constant $C_{p}>0$ such that

$$
\|y\|_{W^{1, p}(\Omega)} \leq C_{p}\left(\|f\|_{L^{1}(\Omega)}+\|u\|_{\mathcal{M}(\Gamma)}\right) .
$$

As a consequence of this theorem, we have that the functional $J_{\Gamma}: \mathcal{M}(\Gamma) \rightarrow \mathbb{R}$ is well defined. Moreover, it is continuous and strictly convex. Therefore, it has a unique minimizer that hereafter will be denoted by $\bar{u}$ with associated optimal state $\bar{y}$. Analogously to Theorem 2.1, if we denote the adjoint state associated to $\bar{u}$ by $\bar{\varphi}$,

$$
\left\{\begin{aligned}
-\Delta \bar{\varphi}+c_{0} \bar{\varphi} & =\bar{y}-y_{d} & & \text { in } \Omega, \\
\partial_{\nu} \bar{\varphi} & =0 & & \text { on } \Gamma,
\end{aligned}\right.
$$

then the following identities hold:

$$
\begin{gathered}
\alpha\|\bar{u}\|_{\mathcal{M}(\Gamma)}+\int_{\Gamma} \bar{\varphi} d \bar{u}=0, \\
\|\bar{\varphi}\|_{C(\Gamma)} \begin{cases}=\alpha & \text { if } \bar{u} \neq 0, \\
\leq \alpha & \text { if } \bar{u}=0 .\end{cases}
\end{gathered}
$$

Then, (5.2) and (5.3) imply a sparsity structure of $\bar{u}$ analogous to (2.7).

To carry out the numerical analysis of problem (1.1), we consider the same triangulation as in section 3. On this triangulation we define the space of discrete states by

$$
Y_{h}=\left\{y_{h} \in C(\bar{\Omega}): y_{h \mid T} \in \mathcal{P}_{1} \text { for every } T \in \mathcal{T}_{h}\right\}
$$

and the discrete state equation

$$
a\left(y_{h}, z_{h}\right)=\int_{\Omega} f z_{h} d x+\int_{\Gamma} z_{h} d u \quad \forall z_{h} \in Y_{h} .
$$

The approximation of the Neumann control problem results in

$$
\left(\mathrm{P}_{\Gamma, h}\right) \quad \min _{u \in \mathcal{M}(\Gamma)} J_{h}\left(u_{h}\right)=\frac{1}{2}\left\|y_{h}-y_{d}\right\|_{L^{2}(\Omega)}^{2}+\alpha\|u\|_{\mathcal{M}(\Gamma)},
$$

where $y_{h}$ is the solution to (5.4). Before analyzing this problem, let us prove the following error estimates concerning the discretization of the state equation. 
Theorem 5.2. Given $u \in \mathcal{M}(\Gamma)$, let $y$ and $y_{h}$ be the solutions to (5.1) and (5.4). Then, there exists a constant $C>0$ independent of $h, f$, and $u$ such that

$$
\left\|y-y_{h}\right\|_{L^{2}(\Omega)} \leq C h^{\kappa}\left(\|f\|_{L^{1}(\Omega)}+\|u\|_{\mathcal{M}(\Gamma)}\right)
$$

with $\kappa$ as in Theorem 4.1.

Proof. Here we follow the lines of the proof [2, Theorem 3]. For any function $g \in L^{2}(\Omega)$, let $z \in H^{2}(\Omega)$ be the solution to

$$
\left\{\begin{aligned}
-\Delta z+c_{0} z=g & \text { in } \Omega \\
\partial_{\nu} z=0 & \text { on } \Gamma
\end{aligned}\right.
$$

and $z_{h} \in Y_{h}$ the solution to

$$
a\left(z_{h}, \phi_{h}\right)=\int_{\Omega} g \phi_{h} d x \quad \forall \phi_{h} \in Y_{h}
$$

Using Green's formula, we obtain

$$
\begin{aligned}
\int_{\Omega} g\left(y-y_{h}\right) d x & =a\left(y-y_{h}, z\right)=a(y, z)-a\left(y_{h}, z\right)=a(y, z)-a\left(y_{h}, z_{h}\right) \\
& =\int_{\Omega} f\left(z-z_{h}\right) d x+\int_{\Gamma}\left(z-z_{h}\right) d u \\
& \leq\left(\|f\|_{L^{1}(\Omega)}+\|u\|_{\mathcal{M}(\Gamma)}\right)\left\|z-z_{h}\right\|_{\infty} \\
& \leq C\left(\|f\|_{L^{1}(\Omega)}+\|u\|_{\mathcal{M}(\Gamma)}\right) h^{\kappa}\|z\|_{H^{2}(\Omega)} \\
& \leq C\left(\|f\|_{L^{1}(\Omega)}+\|u\|_{\mathcal{M}(\Gamma)}\right) h^{\kappa}\|g\|_{L^{2}(\Omega)}
\end{aligned}
$$

where we have used the classical finite element error estimate; see, for instance, [5, Chapter 3]. Since $g \in L^{2}(\Omega)$ is arbitrary, this gives the desired estimate.

Analogously to section 3 , we will denote by $\left\{x_{j}\right\}_{j=1}^{M(h)}$ the boundary nodes of the triangulation $\mathcal{T}_{h}$. Associated to these nodes we consider the space

$$
Y_{h}^{\Gamma}=\left\{y_{h} \in C(\Gamma): y_{h \mid T \cap \Gamma} \in \mathcal{P}_{1}(T \cap \Gamma) \text { for every } T \in \mathcal{T}_{h}^{\Gamma}\right\},
$$

where $\left\{\mathcal{T}_{h}^{\Gamma}\right\}_{h>0}$ is the family of boundary triangles. A nodal basis of $Y_{h}^{\Gamma}$ is given by the functions $\left\{e_{j}\right\}_{j=1}^{M(h)}$ such that $e_{j}\left(x_{i}\right)=\delta_{i j}$ for every $1 \leq i, j \leq M(h)$. Then, every element $y_{h}$ of $Y_{h}^{\Gamma}$ can be written in the form

$$
y_{h}=\sum_{j=1}^{M(h)} y_{j} e_{j}, \quad \text { where } \quad y_{j}=y_{h}\left(x_{j}\right), 1 \leq j \leq M(h) .
$$

We also consider the space

$$
D_{h}^{\Gamma}=\left\{u_{h} \in \mathcal{M}(\Gamma): u_{h}=\sum_{j=1}^{M(h)} \lambda_{j} \delta_{x_{j}}, \text { where }\left\{\lambda_{j}\right\}_{j=1}^{M(h)} \subset \mathbb{R}\right\} .
$$

Above, $\delta_{x_{j}}$ denotes the Dirac measure centered at the node $x_{j}$. It is obvious that $D_{h}^{\Gamma}$ can be identified with the dual of $Y_{h}^{\Gamma}$ through the duality relation

$$
\left\langle u_{h}, y_{h}\right\rangle=\sum_{j=1}^{M(h)} \lambda_{j} y_{j}
$$

Copyright $\odot$ by SIAM. Unauthorized reproduction of this article is prohibited. 
Now, we define the linear operators $\Pi_{h}: C(\Gamma) \rightarrow Y_{h}^{\Gamma}$ and $\Lambda_{h}: \mathcal{M}(\Gamma) \rightarrow D_{h}^{\Gamma}$ by

$$
\Pi_{h} y=\sum_{j=1}^{M(h)} y\left(x_{j}\right) e_{j} \quad \text { and } \quad \Lambda_{h} u=\sum_{j=1}^{M(h)}\left\langle u, e_{j}\right\rangle \delta_{x_{j}} .
$$

With the above notation, the identities (3.4) and (3.5) remain valid and (3.6) and (3.7) hold with $\Omega$ replaced by $\Gamma$. Also, statement 4 of Theorem 3.1 remains correct for $u \in \mathcal{M}(\Gamma)$. This, in particular, implies that Theorem 3.2 remains valid for the case of Neumann boundary control.

The analogous inequalities to (3.8) and (3.9) are

$$
\begin{aligned}
& \left\|u-\Lambda_{h} u\right\|_{W^{-\frac{1}{p}, p}(\Gamma)} \leq C h^{1-n / p^{\prime}}\|u\|_{\mathcal{M}(\Gamma)}, \quad 1<p<\frac{n}{n-1}, \\
& \left\|u-\Lambda_{h} u\right\|_{W^{1, \infty}(\Gamma)^{*}} \leq C h\|u\|_{\mathcal{M}(\Gamma)} .
\end{aligned}
$$

To prove these inequalities let us consider an arbitrary element $z \in W^{\frac{1}{p}, p^{\prime}}(\Gamma)$. It is well known that $W^{\frac{1}{p}, p^{\prime}}(\Gamma)$ is the trace space of $W^{1, p^{\prime}}(\Omega) \subset C(\bar{\Omega})$; see [11]. Given $w \in W^{1, p^{\prime}}(\Omega)$, let us denote by $w_{h}$ its nodal interpolation on the triangulation of $\bar{\Omega}$. Then, arguing as in section 3 , we obtain

$$
\begin{aligned}
\left\langle u-\Lambda_{h} u, z\right\rangle=\left\langle u, z-\Pi_{h} z\right\rangle & \leq\|u\|_{\mathcal{M}(\Gamma)}\left\|z-\Pi_{h} z\right\|_{C(\Gamma)} \\
& \leq\|u\|_{\mathcal{M}(\Gamma)} \inf _{w \in W^{1, p^{\prime}}(\Omega), \gamma(w)=z}\left\|w-w_{h}\right\|_{C(\bar{\Omega})} \\
& \leq C h^{1-n / p^{\prime}}\|u\|_{\mathcal{M}(\Omega)} \inf _{w \in W^{1, p^{\prime}}(\Omega), \gamma(w)=z}\|w\|_{W_{0}^{1, p^{\prime}}(\Omega)} \\
& =C h^{1-n / p^{\prime}}\|u\|_{\mathcal{M}(\Omega)}\|z\|_{W^{\frac{1}{p}, p^{\prime}}(\Gamma)} .
\end{aligned}
$$

Since $W^{\frac{1}{p}, p^{\prime}}(\Gamma)^{*}=W^{-\frac{1}{p}, p}(\Gamma)$, the inequality (3.11) follows from the above inequality. The inequality (3.12) is proved analogously.

Hereafter, $\bar{u}_{h}$ will denote the unique solution to (3.3) in the space $D_{h}$ with the associated discrete state $\bar{y}_{h}$. Then, as a consequence of Theorem 5.2 and the previous observations, we get that Theorem 3.2 remains true with $\Omega$ replaced by $\Gamma$.

Finally, error estimates analogous to (4.2) and (4.7) can be obtained following the same arguments, replacing (4.3) by (5.5) and taking into account that $\Omega=\Omega_{h}$, which obviously simplifies the proofs.

6. Computational results. We illustrate the theoretical results of the previous sections with numerical examples in two dimensions. For our computational domain, we take the square $\Omega_{h}=\Omega=[-1,1]^{2}$, which is discretized using the standard uniform triangulation arising from $N \times N$ equidistributed nodes. Unless stated otherwise, we fix $N=128$, which corresponds to $h \approx 0.0157, c_{0}=0$, and $\alpha=10^{-2}$.

The numerical solution of the discrete optimality system is based on an equivalent formulation of the optimality conditions (2.3) and (2.4). Returning to the characterization (2.5) of the subgradient, we have that the adjoint state $\bar{\varphi} \in C_{0}(\Omega)$ satisfies

$$
-\bar{\varphi} \in \alpha \partial\|\cdot\|_{\mathcal{M}(\Omega)}(\bar{u}) .
$$

By the definition of the convex subdifferential, this is equivalent to

$$
\bar{u} \in \partial I_{\left\{z \in C_{0}(\Omega):\|z\|_{C_{0}(\Omega)} \leq \alpha\right\}}(-\bar{\varphi}),
$$

since the Fenchel conjugate of the indicator function of the (scaled) unit ball in $C_{0}(\Omega)$ 


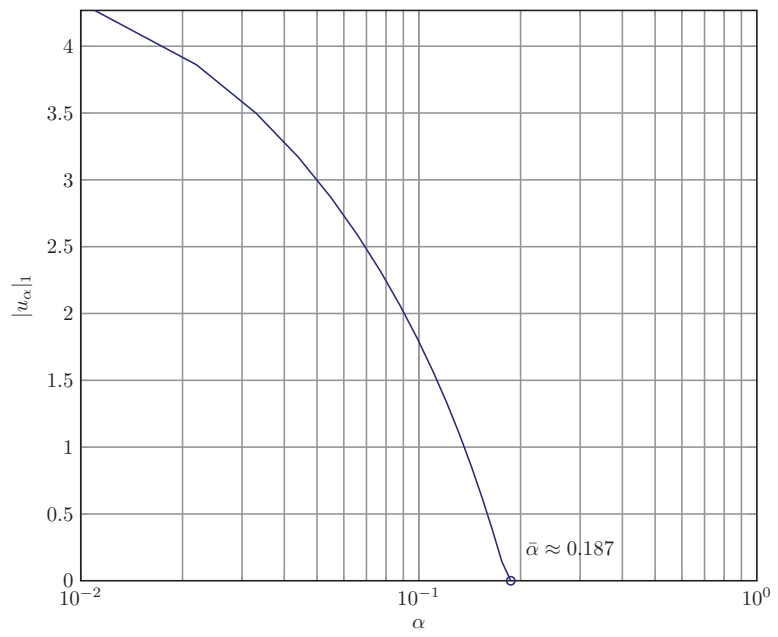

Fig. 6.1. Dependence of norm of optimal control $u_{h}$ on penalty parameter $\alpha$.

is the (scaled) norm in $\mathcal{M}(\Omega)$. The subdifferential of the indicator function is then given by the normal cone, which can be characterized by the variational inequality

$$
\langle\bar{u}, \bar{\varphi}-\varphi\rangle \leq 0 \quad \forall\|\varphi\|_{C_{0}(\Omega)} \leq \alpha .
$$

We now pass to the discrete setting by replacing the continuous control $\bar{u}$ with its discretization $\bar{u}_{h}$ and introducing the discrete adjoint state $\bar{\varphi}_{h}=\sum_{j=1}^{N(h)} \varphi_{j} e_{j} \in Y_{h}$. The above variational inequality can then be reformulated using a complementarity function as

$$
\bar{u}_{h}+\max \left(0,-\bar{u}_{h}+\bar{\varphi}_{h}-\alpha\right)+\min \left(0,-\bar{u}_{h}+\bar{\varphi}_{h}+\alpha\right)=0,
$$

which should be understood componentwise in terms of the vector of expansion coefficients $\left(\lambda_{1}, \ldots, \lambda_{N(h)}\right)$ and $\left(\varphi_{1}, \ldots, \varphi_{N(h)}\right)$. This is a locally Lipschitz mapping from $\mathbb{R}^{N(h)} \times \mathbb{R}^{N(h)} \rightarrow \mathbb{R}^{N(h)}$ and thus the reformulated discrete optimality system can be solved by a locally superlinearly convergent semismooth Newton method [10], [12]. The corresponding algorithm was implemented in MATLAB (R2011a).

We first illustrate the structural properties of the optimal controls. Figure 6.1 shows the norm of the optimal control $u_{\alpha}$ as a function of the penalty parameter $\alpha$. As verified in Proposition 2.2, there exists an $\bar{\alpha}(\approx 0.187)$, such that $u_{\alpha} \equiv 0$ for $\alpha>\bar{\alpha}$.

The statement of Proposition 2.3 is illustrated in Figure 6.2, where the optimal controls for the target $y_{d}=10 \exp \left(-50\|x\|^{2}\right)$ and different observation domains $\omega_{y}$ are compared. As a reference, Figure 6.2(a) shows the control for $\omega_{y}=\Omega$ (in the form of its expansion coefficients $\lambda_{j}$ at each grid point, with linear interpolation for better visibility). In contrast, the control for $\omega_{y}=\chi_{\left\{\left|x_{1}\right|<1 / 2\right\}} \chi_{\left\{\left|x_{2}\right|<1 / 4\right\}} \subsetneq \Omega$ vanishes outside of $\omega_{y}$; see Figure 6.2(b).

We now investigate the convergence behavior as $h \rightarrow 0$. In the absence of a known exact solution, we take as a reference solution the computed optimal discrete control and optimal discrete state on the finest grid with $N^{*}=2^{10}$, corresponding to $h^{*}=$ $2 \cdot 10^{-3}$. We first consider distributed control, with the target $y_{d, 1}$ given in Figure 6.3(a). Figure 6.4(a) shows the difference $\left|J_{h}-J_{h^{*}}\right|$ for a series of successively refined, nested grids for $N=2^{3}, \ldots, 2^{9}$. The observed linear convergence rate agrees well with the 


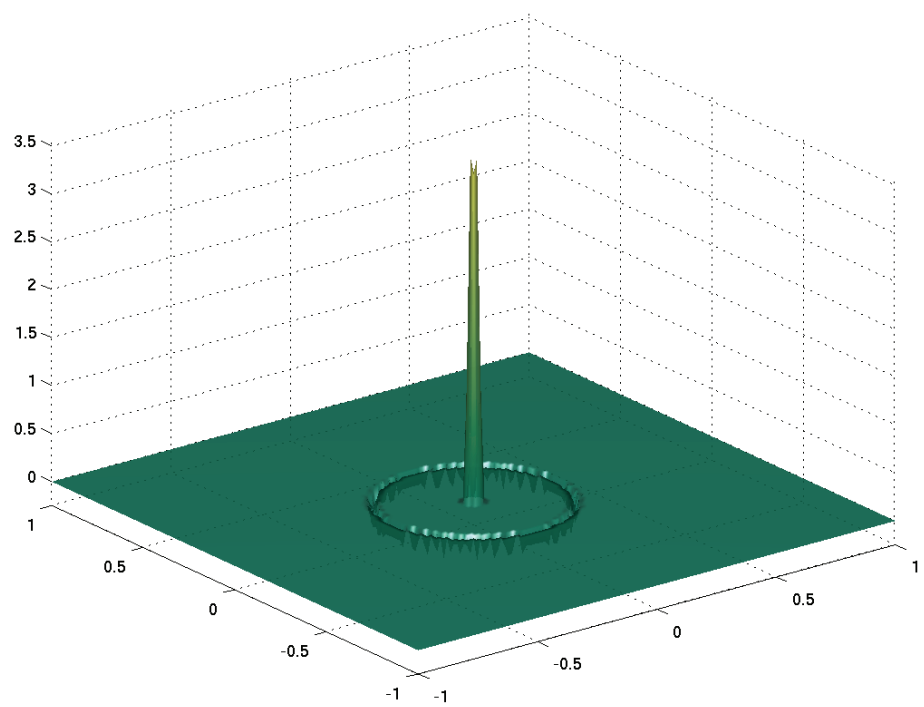

(a) $u_{h}$, observation on $\omega_{y}=\Omega$

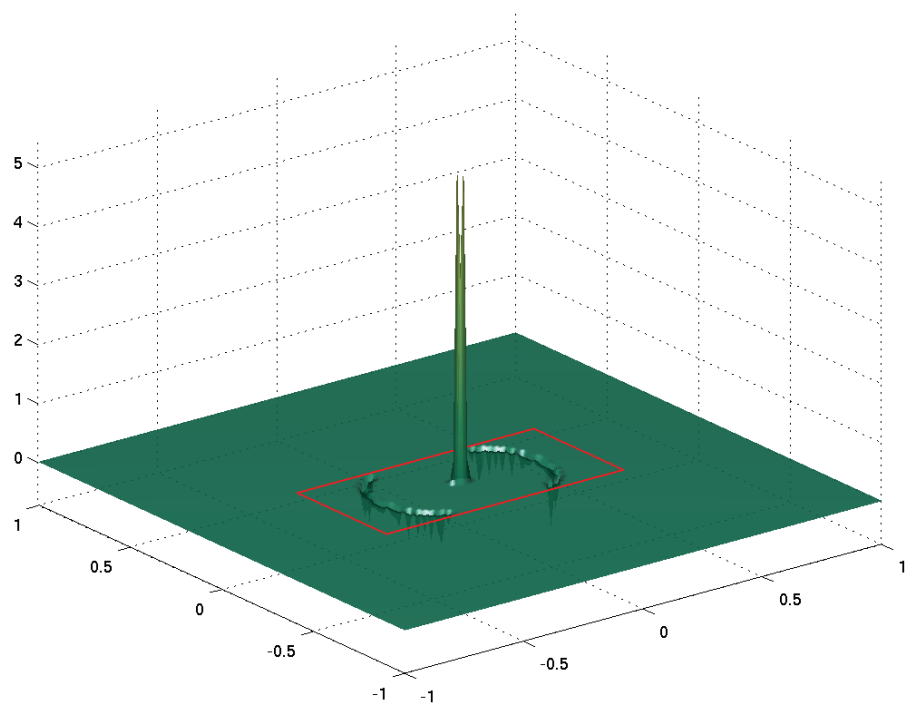

(b) $u_{h}$, observation on $\omega_{y} \subsetneq \Omega$ (in red)

FIG. 6.2. Comparison of optimal controls $u_{h}$ for full observation $\left(\omega_{y}=\Omega\right)$ and partial observation ( $\omega_{y} \subsetneq \Omega$, marked in red). Color is available only in the online version.

rate obtained in Theorem 4.1. The corresponding $L^{2}$ error $\left\|y_{h}-y_{h^{*}}\right\|_{L^{2}}$ of the discrete states also decays with a linear rate, which is faster than predicted by Theorem 4.2.

For the case of Neumann control, we set $\alpha=5 \cdot 10^{-2}$ and $c_{0}=10^{-2}$ and consider the target $y_{d, 2}$ shown in Figure 6.3(b). Again, both the error in the functional value (Figure 6.5(a)) and in the state (Figure 6.5(b)) follow an approximately linear convergence rate. To illustrate the sparsity properties of Neumann boundary controls, Figure 6.6 shows the optimal control $u_{h, \alpha}$ (again, in the form of its linearly interpolated coefficients $\lambda_{j}$ ) for $\alpha=10^{-3}, 10^{-2}$ and $10^{-1}$, plotted along boundary sections as indicated. 


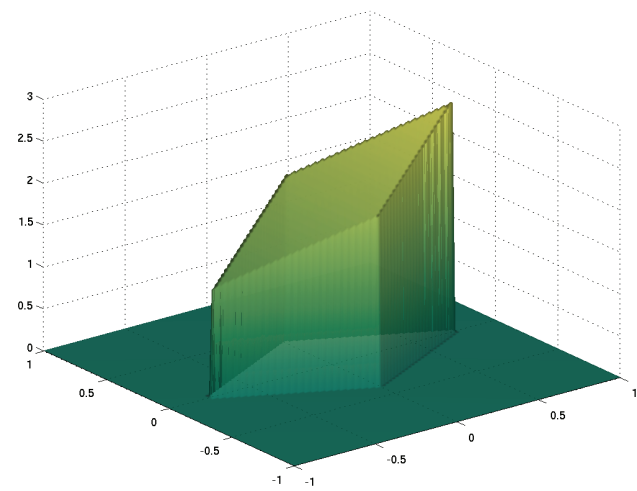

(a) target $y_{d, 1}$

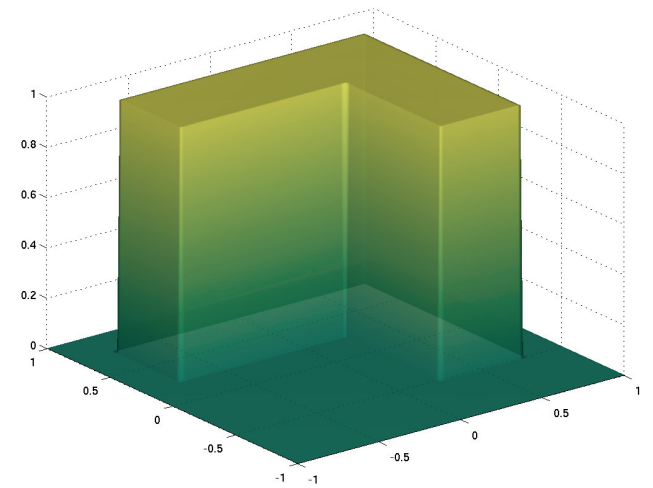

(b) target $y_{d, 2}$

FIG. 6.3. Target states for convergence rate examples.

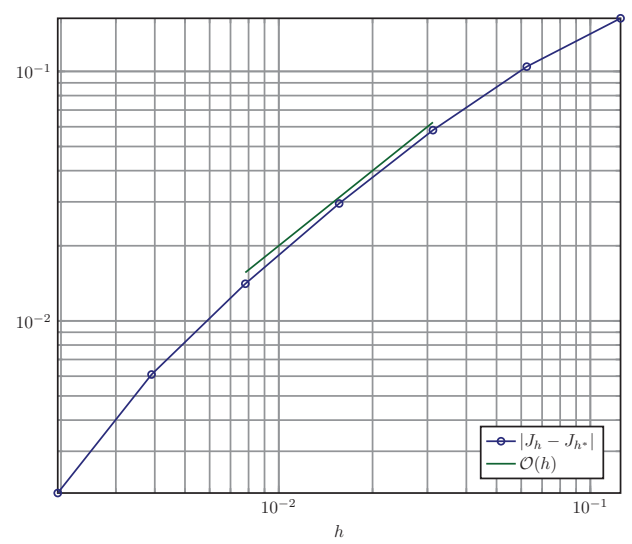

(a) functional value $J_{h}$

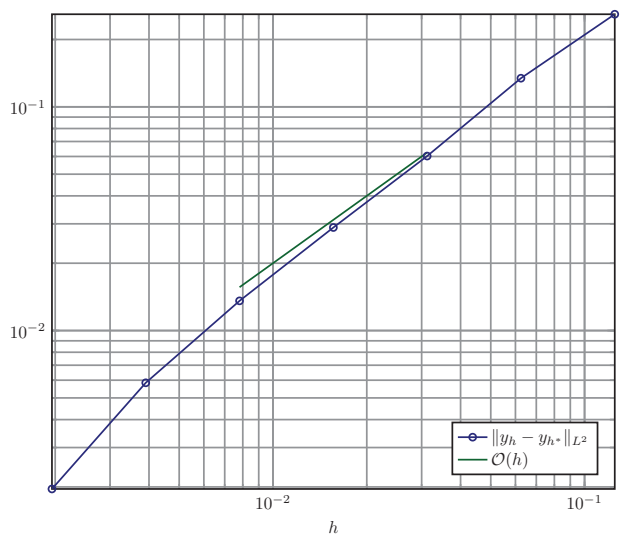

(b) $L^{2}$ norm of state $y_{h}$

FIG. 6.4. Illustration of convergence order for distributed control.

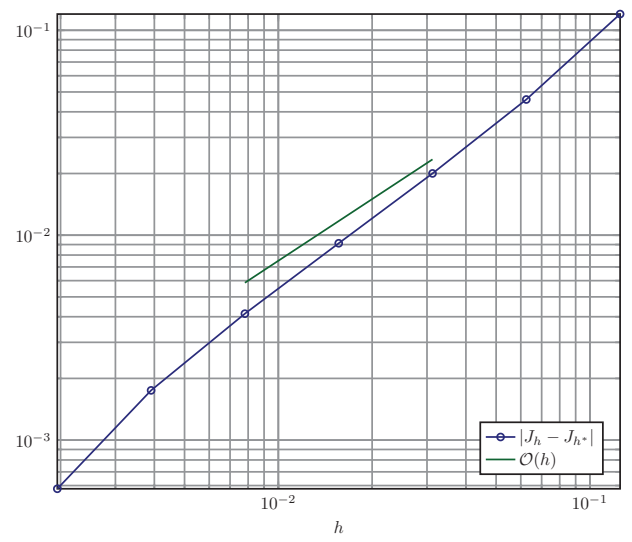

(a) functional value $J_{h}$

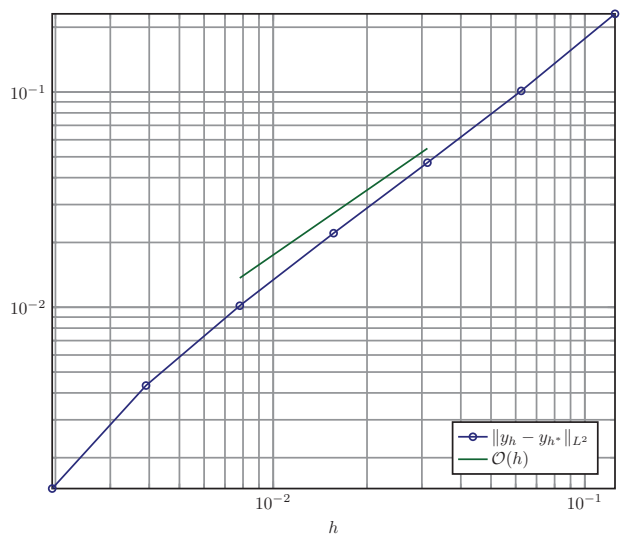

(b) $L^{2}$ norm of state $y_{h}$

FIG. 6.5. Illustration of convergence order for Neumann control. 


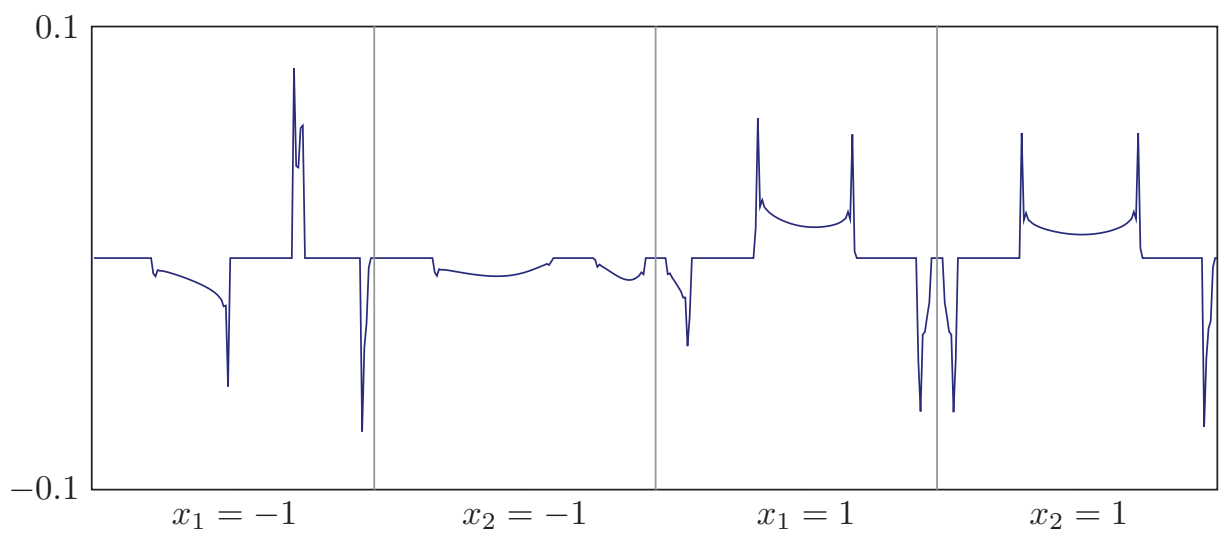

(a) $\alpha=10^{-3}$

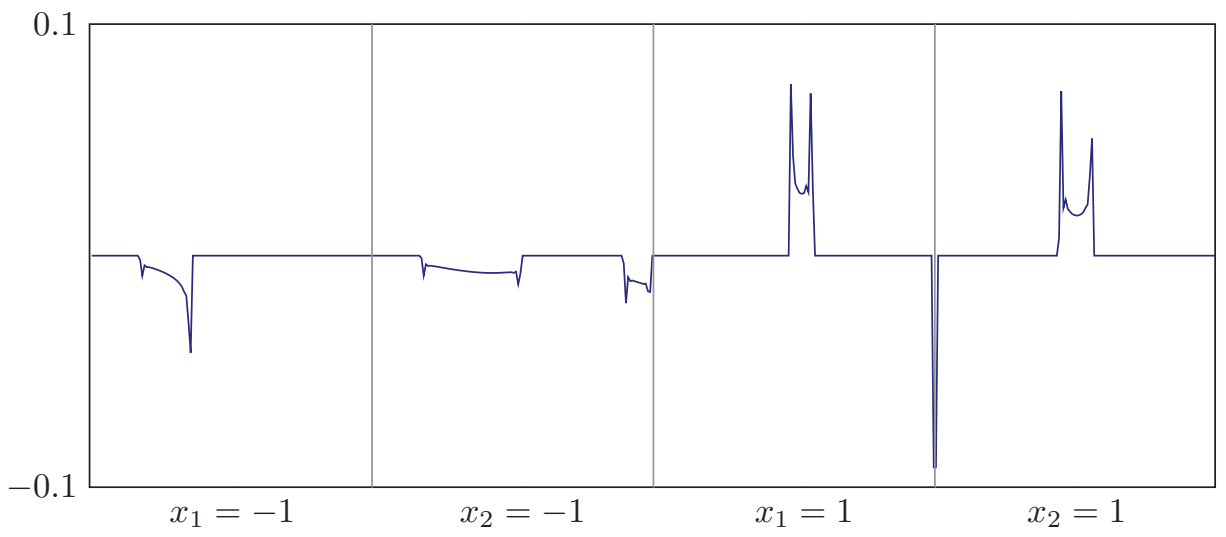

(b) $\alpha=10^{-2}$

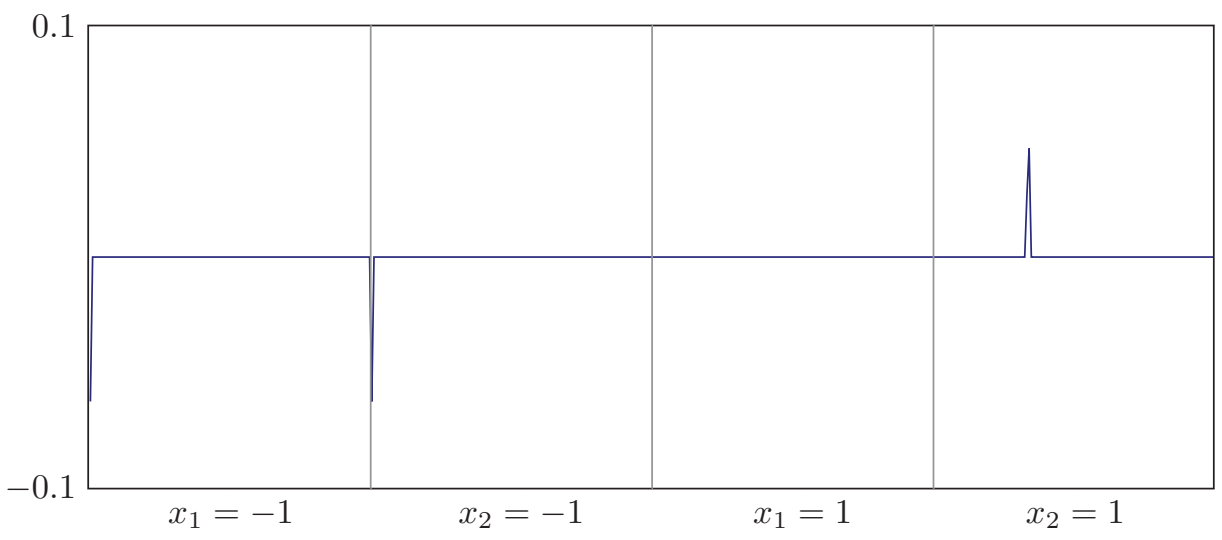

(c) $\alpha=10^{-1}$

FIG. 6.6. Optimal Neumann control $u_{h, \alpha}$ for increasing values of $\alpha$.

Copyright $@$ by SIAM. Unauthorized reproduction of this article is prohibited. 
7. Conclusion. By considering optimal control problems in spaces of measures, controls with strong sparsity properties can be obtained. Although the nonreflexive Banach space setting complicates the analysis, a straightforward numerical approximation that retains the structural properties of the measure norm is possible. In a sense, the results of this paper justify the "intuitive" discretization of regular Borel measures by Dirac measures on a set of nodes.

\section{REFERENCES}

[1] K. Bredies and H. Pikkarainen, Inverse problems in spaces of measures, ESAIM Control Optim. Calc. Var., 2012, DOI: 10.1051/cocv/2011205.

[2] E. CASAS, $L^{2}$ estimates for the finite element method for the Dirichlet problem with singular data, Numer. Math., 47 (1985), pp. 627-632.

[3] E. CASAS, Control of an elliptic problem with pointwise state constraints, SIAM J. Control Optim., 24 (1986), pp. 1309-1318.

[4] E. Casas, R. Herzog, and G. Wachsmuth, Optimality conditions and error analysis of semilinear elliptic control problems with $L^{1}$ cost functional, SIAM J. Optim., to appear.

[5] P. G. Ciarlet, The Finite Element Method for Elliptic Problems, North-Holland, Amsterdam, 1978.

[6] C. Clason and K. Kunisch, A duality-based approach to elliptic control problems in nonreflexive Banach spaces, ESAIM Control Optim. Calc. Var., 17 (2011), pp. 243-266.

[7] C. Clason and K. Kunisch, A measure space approach to optimal source placement, Comput. Optim. Appl., (2011), DOI 10.1007/s10589-011-9444-9.

[8] M. HINZE, A variational discretization concept in control constrained optimization: The linearquadratic case, Comput. Optim. Appl., 30 (2005), pp. 45-61.

[9] D. Jerison and C. Kenig, The inhomogeneous Dirichlet problem in Lipschitz domains, J. Funct. Anal., 130 (1995), pp. 161-219.

[10] B. Kummer, Newton's method based on generalized derivatives for nonsmooth functions: Convergence analysis, in Advances in Optimization (Lambrecht, 1991), Lecture Notes in Econom. and Math. Systems 382, Springer, Berlin, 1992, pp. 171-194.

[11] J. NeČAs, Les Méthodes Directes en Théorie des Equations Elliptiques, Editeurs Academia, Prague, 1967.

[12] L. Qi And J. Sun, A nonsmooth version of Newton's method, Math. Program., 58 (1993), pp. 353-367.

[13] P.-A. Raviart And J.-M. Thomas, Introduction à L'analyse Numérique des Equations aux Dérivées Partielles, Masson, Paris, 1983.

[14] W. Rudin, Real and Complex Analysis, McGraw-Hill, London, 1970.

[15] G. STADLER, Elliptic optimal control problems with $L^{1}$-control cost and applications for the placement of control devices, Comput. Optim. Appl., 44 (2009), pp. 159-181.

[16] G. Wachsmuth AND D. WaChSmuth, Convergence and regularisation results for optimal control problems with sparsity functional, ESAIM Control Optim. Calc. Var., (2010), DOI: $10.1051 / \mathrm{cocv} / 2010027$.

Copyright $@$ by SIAM. Unauthorized reproduction of this article is prohibited. 